$\mathrm{O}-\mathrm{\square} \mathrm{C}$

\title{
Chemical-state-discriminated hard X-ray photoelectron diffraction study for polar InN
}

\author{
Yoshiyuki Yamashita ${ }^{1}$, Anli Yang ${ }^{1}$, and Keisuke Kobayashi ${ }^{2}$ \\ ${ }^{1}$ National Institute for Materials Science, 1-1 Namiki, Tsukuba, 305-0044, Japan \\ ${ }^{2}$ Japan Atomic Energy Agency, 1-1-1 Koto, Sayo, 679-5148, Japan \\ *corresponding author’s e-mail: yamashita.yoshiyuki@nims.go.jp
}

(Received: May 16, 2019; Accepted: July 10, 2019)

\begin{abstract}
Near-surface structures of polar InN films were investigated by laboratory-based hard x-ray photoelectron diffraction (HXPD) with chemical-state-discrimination. HXPD patterns from In $3 \mathrm{~d}_{5 / 2}$ and $\mathrm{N} 1 \mathrm{~s}$ core levels of the In-polar and Npolar InN films were different from each other and compared with the simulation results using a multiple-scattering cluster model. It was found that the near-surface structure of the In-polar InN film was close to the ideal wurtzite structure. On the other hand, on the N-polar InN film, defects-rich surface was formed. In addition, the existence of the In-polar domains was observed in the HXPD patterns.
\end{abstract}

\section{Introduction}

With the small band gap $(\sim 0.7 \mathrm{eV})$ and superior electrical transport properties, $\mathrm{InN}$ is attracting great attention for potential region optoelectronic devices such as emitters spanning a wider wavelength range and high-efficiency photovoltaic devices.[1] For InN single crystal, as a wurtzite crystal lacks inversion symmetry along the c-axis direction, In-polar and N-polar InN exhibit different properties. For example, the maximum growth temperature for $\mathrm{N}$-polar $\mathrm{InN}$ is about $100{ }^{\circ} \mathrm{C}$ higher than In-polar InN. The N-polar InN shows better bulk crystalline quality. However, an atomically flat surface can be observed for the In-polar InN but not for the N-polar one yet.[2]

The critical issue of $\mathrm{InN}$ is that $\mathrm{PN}$ junction has not been realized yet. Therefore, in order to fully realize the potential of $\mathrm{InN}$, a fundamental understanding of structures near surface are required. For the near surface structures, photoelectron diffraction (PED) is powerful tool to investigate the structures. In addition, PED is the element and chemical state specificity so as to elucidate a local atomic structure. [3] Hard x-ray photoelectron diffraction (HXPD) is expected to open up additional analytical possibilities for materials because of the larger probing depth. In this study, we employed high energy angle resolved spectroscopy for laboratory (HEARP Lab, hv=5414.7 eV). [4] HEARP provides hard $\mathrm{x}$-ray photoelectron spectroscopy (HAXES) and HXPD, which enables us to elucidate local atomic structures. In the present study, we have investigated near surface structures of polar InN using HEARP lab.

\section{Experimental}

Two kinds of InN films were prepared by radiofrequency plasma-assisted MBE. For In-polar, in situ In droplet elimination by radical beam irradiation was employed. An N-polar InN film with a thickness of about $750 \mathrm{~nm}$ was grown directly on a nitrided sapphire. The two samples were cleaned using acetone, methanol, $35 \%$ hydrochloric acid, and deionized water before measurement.

The HXPD system consists of a monochromatic $\mathrm{Cr}$ $\mathrm{K} \alpha$ source ( $5414.7 \mathrm{eV}$ ), a high energy version of the VG SCIENTA R4000 $10 \mathrm{kV}$ analyzer with wide acceptance objective lens. The angle acceptance of the combined objective lens and the analyzer is $\pm 35^{\circ}$ with an angular resolution of $0.5^{\circ}$. The total energy resolution was $1 \mathrm{eV}$. In this study, the polar angle $\theta$ is defined to be zero for the photoemission direction normal to a sample surface. 


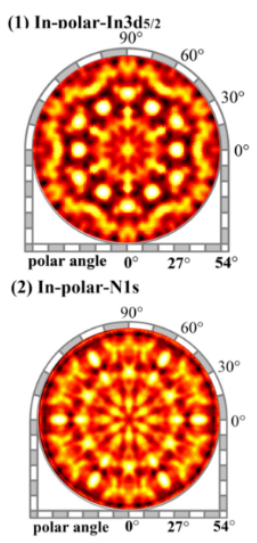

Experiment

(3) N-polar-In3ds/2

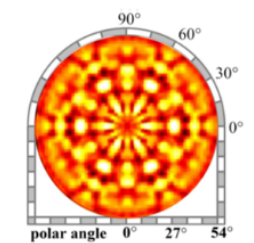

(4) N-polar-N1s

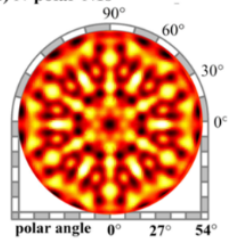

Experiment
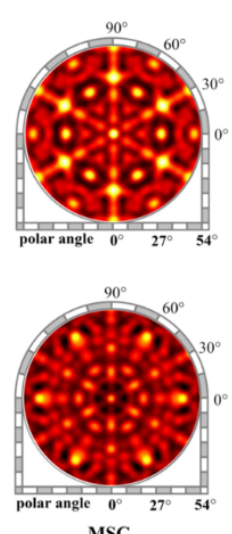

MSC
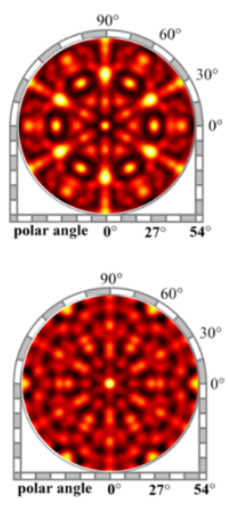

MSC
Fig.1 Experimental (left) and multiple scattering cluster (MSC) calculations (right) HXPD patterns

The $\theta$-dependent sensitivity of the analyzer, was calibrated using an amorphous GeSbTe sample.

\section{Results and discussion}

Figure 1 shows HXPD patterns of In-polar and Npolar InN. For structural analysis, we found that the diffraction patterns were dominated by forward scattering of nearest-neighbor In atoms. This is because the strong forward focusing was dominant in this energy range.

In the case of In-polar InN, In $3 \mathrm{~d}_{5 / 2}$ pattern to (1010) M-plane ((11-20) A-plane) exhibited the strong spots of $30^{\circ}\left(17^{\circ}\right), 40^{\circ}\left(30^{\circ}\right)$, and $50^{\circ}\left(40^{\circ}\right)$ while $\mathrm{N}$ 1s patterns in M plane (A plane) for In-polar InN exhibited the strong spots of $20^{\circ}, 30^{\circ}\left(30^{\circ}\right)$, and $45^{\circ}\left(45^{\circ}\right)$. These patterns were good agreements with the diffraction patterns of the ideal wurtzite structure.

In the case of N-polar InN, two components for both In $3 \mathrm{~d}_{5 / 2}$ and $\mathrm{N} 1 \mathrm{~s}$ existed. the chemical-statediscriminated N-polar In $3 \mathrm{~d}_{5 / 2}$ and N1s HXPD patterns indicated that main component of the core levels exhibited clear diffraction patters. On the other hand, the patters of the satellite component were unclear. Angle resolved core levels analysis indicated that main component was come from bulk while the satellite component was surface origin. Thus, the surface exhibited defect rich and/or amorphous structure. Comparison of the main components diffraction patterns with the simulation results based on a multiplescattering cluster model exhibited an additional structure. We found that N-polar InN may be mixed with In-polar InN domain in a near-surface area of the $\mathrm{N}$-polar InN film. Thus, in the N-polar InN film, the existence of In-polar domains in the near surface region was observed, and the defect atoms of N-polar InN film were located on the surface.

\section{References}

[1] Y. Nanishi, Y. Saito, and T. Yamaguchi, Jpn. J. Appl. Phys. 42, 2549 (2003).

[2] K. Xu and A. Yoshikawa, Appl. Phys. Lett. 83, 251 (2003).

[3] C. S. Fadley, M. A. Van Hove, Z. Hussain, and A. P. Kaduwela, J. Electron Spectrosc. Relat. Phenom. 75, 273 (1995).

[4] M. Kobata, I. Pis, H. Iwai, H. Yamazui, H. Takahashi, M. Suzuki, H. Matsuda, H. Daimon, and K. Kobayashi, Anal. Sci. 26, 227 (2010). 